\title{
Medically Tailored Meal Delivery for Diabetes Patients with Food Insecurity: a Randomized Cross-over Trial
}

\author{
Seth A. Berkowitz, MD MPH ${ }^{1,2,3,4}$, Linda M. Delahanty, MS RDN 2,3, Jean Terranova, JD",

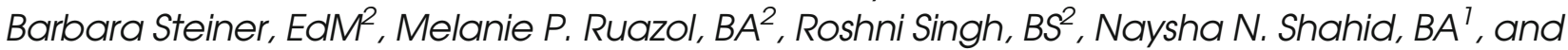 \\ Deborah J. Wexler, MD MSc ${ }^{2,3}$
}

\begin{abstract}
'Division of General Internal Medicine, Massachusetts General Hospital, Boston, MA, USA; ${ }^{2}$ Diabetes Unit, Massachusetts General Hospital, Boston, MA, USA; ${ }^{3}$ Harvard Medical School, Boston, MA, USA; ${ }^{4}$ Division of General Medicine and Clinical Epidemiology, Department of Medicine, University of North Carolina School of Medicine, Chapel Hill, NC, USA; ${ }^{5}$ Community Servings, INC, Jamaica Plain, MA, USA.
\end{abstract}

BACKGROUND: Food insecurity, defined as inconsistent food access owing to cost, leads to poor health.

OBJECTIVE: To test whether a medically tailored meal delivery program improved dietary quality in individuals with type 2 diabetes and food insecurity.

DESIGN: Randomized cross-over clinical trial.

PARTICIPANTS: Forty-four adults with diabetes, hemoglobin Alc > 8.0\%, and food insecurity (defined as at least one positive item on the two-item "Hunger Vital Sign").

INTERVENTION: In the Community Servings: Food as Medicine for Diabetes cross-over clinical trial (NCT02426138), conducted from June 2015 to July 2017, we randomly assigned the order of "on-meals" (home delivery of 10 meals/week for 12 weeks delivered by Community Servings, a non-profit organization) and "offmeals" (12 weeks usual care and a Choose MyPlate healthy eating brochure) periods.

MAIN MEASURES: The primary outcome was Healthy Eating Index 2010 score (HEI), assessed by three 24$\mathrm{h}$ food recalls in both periods. Higher HEI score (range 0-100; clinically significant difference 5) represents better dietary quality. Secondary outcomes included food insecurity and self-reported hypoglycemia.

KEY RESULTS: Mean “on-meal” HEI score was 71.3 (SD 7.5) while mean "off-meal" HEI score was 39.9 (SD 7.8) (difference 31.4 points, $p<0.0001$ ). Participants experienced improvements in almost all sub-categories of HEI score, with increased consumption of vegetables, fruits, and whole grains and decreased solid fats, alcohol, and added sugar consumption. Participants also reported lower food insecurity (42\% "on-meal" vs. 62\% "off-meal," $p=0.047$ ), less hypoglycemia (47\% “on-meal” vs. $64 \%$ "off-meal," $p=0.03$ ), and fewer days where mental health interfered with quality of life (5.65 vs. 9.59 days out of 30 , $p=0.03)$.

CONCLUSIONS: For food-insecure individuals with diabetes, medically tailored meals improved dietary quality and food insecurity and reduced hypoglycemia. Longer-

Electronic supplementary material The online version of this article (https://doi.org/10.1007/s11606-018-4716-z) contains supplementary material, which is available to authorized users.

Received April 19, 2018

Revised August 30, 2018

Accepted October 15, 2018

Published online November 12, 2018 term studies should evaluate effects on diabetes control (e.g., hemoglobin Alc) and patient-reported outcomes (e.g., well-being).

KEY WORDS: type 2 diabetes mellitus; food insecurity; dietary quality; hypoglycemia.

J Gen Intern Med 34(3):396-404

DOI: $10.1007 / \mathrm{s} 11606-018-4716-\mathrm{Z}$

(C) Society of General Internal Medicine 2018

$\mathrm{O}$ ne in 10 adult Americans - currently 29 million-has diabetes mellitus. ${ }^{1}$ Type 2 diabetes, which accounts for $95 \%$ of all cases, can be deadly, substantially lowers quality of life, and is responsible for $\$ 245$ billion in annual costs. ${ }^{1}$ However, the burden of diabetes is not distributed equally. Socioeconomically vulnerable patients suffer disproportionately from diabetes complications and mortality. ${ }^{2} \mathrm{~A}$ major contributor to this is food insecurity, defined as limited access to nutritious food owing to cost. ${ }^{3}$ Approximately 20 to $30 \%(6$ to 9 million) of Americans with diabetes report food insecurity each year. ${ }^{4,5}$ A healthy diet is the cornerstone of diabetes management ${ }^{6}$, but food insecurity incents the consumption of inexpensive, calorie-dense food of little nutritional value. ${ }^{7}$ In particular, worse dietary quality among individuals with diabetes and food insecurity, as assessed by the Healthy Eating Index 2010 (HEI 2010) score, is associated with worse glycemic control and increased diabetes complications. ${ }^{8}$ Further, food insecurity is associated with increased risk of hypoglycemia, a serious adverse event related to diabetes therapy. ${ }^{9-12}$

Hyperglycemia is particularly responsive to dietary changes, ${ }^{13,14}$ but previous dietary interventions often emphasize behavior changes that individuals with food insecurity are unlikely to be able to make. Further, clinicians currently have few options for helping patients with food insecurity in routine care, and there is little evidence regarding whether clinical programs for individuals with food insecurity can improve diet. ${ }^{15}$

To address these research gaps, we sought to test the feasibility and short-term impact on diet of a program that 
delivered medically tailored meals to individuals with type 2 diabetes, food insecurity, and hyperglycemia (hemoglobin Alc $>8.0 \%$ ) in a randomized cross-over trial. Because our intervention was specifically designed to address food insecurity, as well as supporting dietary changes, we hypothesized that receiving medically tailored meals would improve the Healthy Eating Index 2010 (HEI) dietary quality score and improve food insecurity.

\section{METHODS}

\section{Study Design}

The Community Servings: Food as Medicine for Diabetes clinical trial used a 24-week randomized cross-over design with no washout period (eFigure 1). We included adults (age $>$ 18 years) with type 2 diabetes, a hemoglobin A1c $>8.0 \%$ in the last year, and who reported food insecurity as assessed by the two-item "hunger vital sign."16, 17 Participants also had to live in the delivery service area (which covered most of eastern Massachusetts) and be able to store and prepare meals. Exclusion criteria included participating in another diabetes research intervention, pregnancy, and food allergies that would prevent participating in the meal program. Participants were drawn from local primary care networks in the Boston metropolitan area (Fig. 1). We conducted outreach for participant recruitment and also accepted referrals from primary care providers. Participants were provided reimbursement for transportation expenses and a small incentive for attending study visits. The meal program was provided at no cost to the participant.

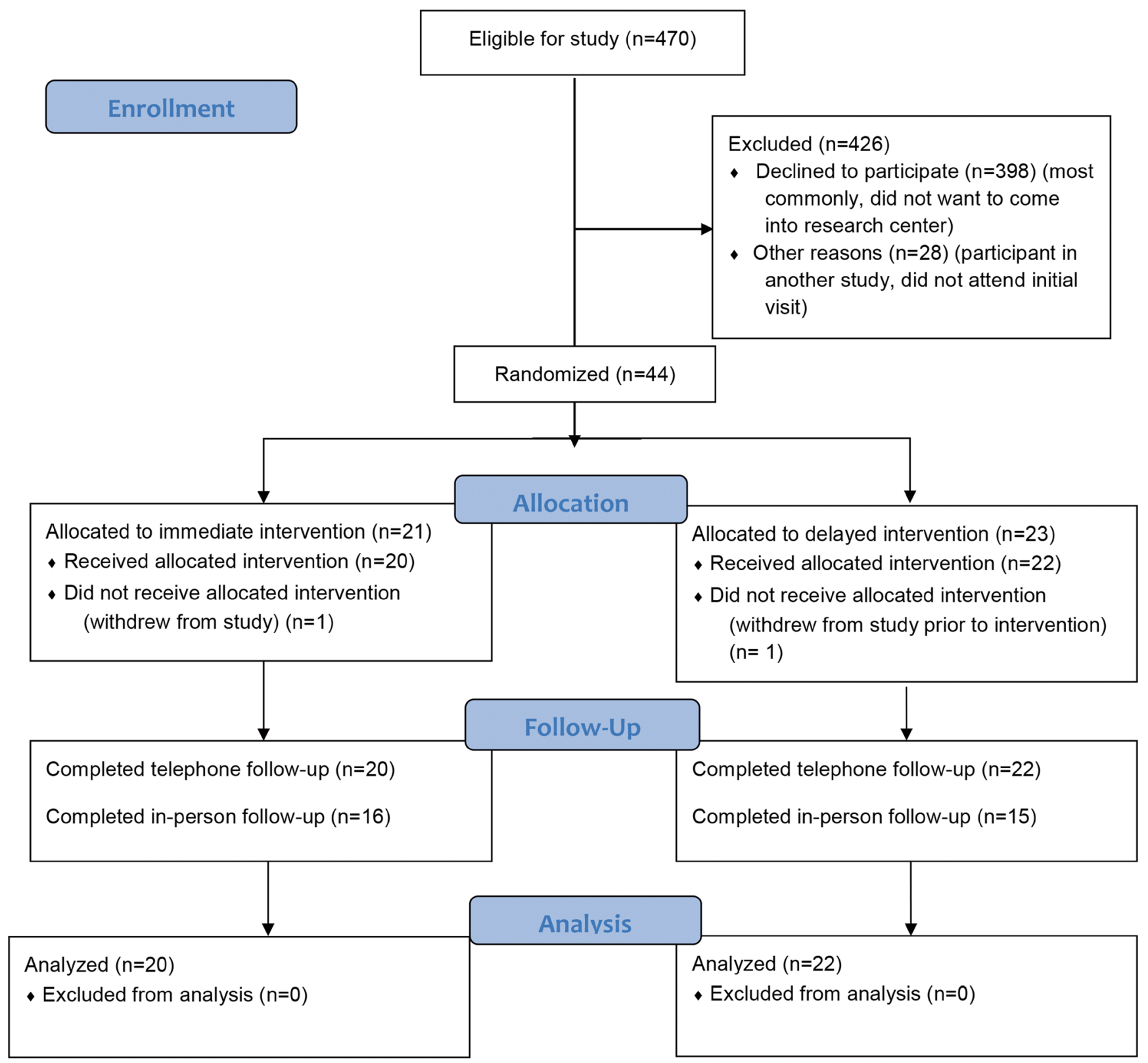

Fig. 1 CONSORT diagram. 
Participants were enrolled from June 1, 2015, through January 31,2017 , and were followed for 24 weeks from enrollment. All data collection was completed by July 31, 2017.

After completing an IRB-approved informed consent process, enrolled participants completed a baseline examination and were then randomly assigned to either immediate receipt of meal delivery for 12 weeks, followed by 12 weeks of follow-up while not receiving the intervention, or delayed receipt of the intervention for 12 weeks, followed by 12 weeks of meal delivery. Randomization was conducted using an electronic random number generator in a variable size block randomized pattern, in a 1:1 ratio between arms. After randomization, intervention assignment was not masked.

This study was approved by the Human Research Committee at Partners Health Care and prospectively registered on ClinicalTrials.gov as NCT02426138.

\section{Intervention}

The intervention in the study consisted of home delivery of medically tailored meals by Community Servings, a local nonprofit organization. Meals were prepared under the guidance of a registered dietitian to be suitable not only for the participant's diagnosis of diabetes but also for other medical conditions the participant might have across 17 possible "tracks" (e.g., chronic kidney disease or anticoagulation using warfarin). Each individual could receive meals that followed up to 3 "tracks" (e.g., diabetes and chronic kidney disease and anticoagulation using warfarin), allowing for meals closely tailored to the specific medical needs of the individual. While receiving the intervention, the participant received a onceweekly delivery consisting of 10 refrigerated and/or frozen meals, designed to represent 5 lunches and 5 dinners (approximately half of the participant's weekly food intake). Meals were fully prepared and could be simply heated and consumed. Food was provided only for the study participant. To test the impact of meal delivery, no additional educational intervention was included during the "on-meals" period.

During the control portion of the study, participants received usual care, plus the Choose My Plate healthy eating brochure.

\section{Outcomes}

The primary goal of this study was to determine whether receipt of medically tailored meals would lead to changes in dietary pattern that would be expected to improve clinical outcomes in a longer-term study. Therefore, the primary outcome in the study was the difference in Healthy Eating Index 2010 (HEI 2010) total score between the "on-meals" and "off-meals" period. The HEI 2010 score ranges from 0 to 100, with higher scores representing a "better" diet that more closely follows guideline recommendations. ${ }^{18}$ Higher HEI 2010 scores have been associated with better hemoglobin A1c trajectories in individuals with diabetes ${ }^{8}$, and with improved cardiovascular morbidity and mortality overall. ${ }^{19}$ The total HEI score is calculated by summing the subscores in 13 categories (score range in parentheses): total vegetables $(0-5)$, greens and beans $(0-5)$, total fruit $(0-5)$, whole fruit $(0-5)$, whole grains $(0-10)$, total dairy $(0-10)$, total protein $(0-5)$, seafood and plant protein $(0-$ 5 ), fatty acids (0-10), sodium, refined grains (0-10), and "empty" calories from solid fats, alcohol, and added sugars $(0-20) .{ }^{18}$ For each category, scoring is such that a higher score represents "better" dietary quality (e.g., more whole grains or fewer calories from sugar). As an example of scale, 0 points for whole fruit represents consuming 0 cups per $1000 \mathrm{kcal}$ per day, and 5 (maximum) points represents $\geq 0.4$ cups per $1000 \mathrm{kcal}$ per day. ${ }^{18}$ Based on prior literature, we pre-specified a total HEI score change greater than 5 points to be clinically significant. ${ }^{8}$, ${ }^{18}$ We also examined changes in the sub-categories of HEI score as secondary outcomes.

Other secondary outcomes included several patientreported outcomes that we hypothesized would be improved by participating in the meal program. These included food insecurity, self-report of hypoglycemia, health-related quality of life, cost-related medication underuse, diabetes distress, and depressive symptoms (see eTable 1 for descriptions of instruments used for outcome assessment). Given the short time frame of the study and number of participants, we did not expect significant changes in biomarkers or anthropomorphic assessments, but we did collect data on $\mathrm{HbAlc}$, body mass index, blood pressure, and fasting lipids for exploratory purposes. We caution that, for these reasons, this study should not be interpreted as testing hypotheses about changes in biomarkers or anthropomorphic measurements,

Consistent with guidelines regarding assessment of changes in diet in clinical trials, HEI 2010 was assessed by completing three 24-h dietary recalls during each period, and averaging the results within the period, using standard scoring macros. ${ }^{20}$ During each study period, one 24-h recall was conducted during an in-person study visit, and two were conducted over the telephone, at approximately 4 and 8 weeks into the study period. To make an accurate assessment of diet, telephone visits were unscheduled, and we sought to assess both weekday and weekend eating. In addition to the "on-meals" and "off-meals" 24-h recalls, we also conducted a single 24$\mathrm{h}$ recall at the initial study visit.

Secondary outcomes were assessed using validated instruments during in-person study visits (see Supplemental Material for instruments and description of measurement protocols). Anthropomorphic assessments followed standardized study procedures and all used the same equipment. Laboratory samples were analyzed in the same central certified laboratory. The hemoglobin A1c assay used was traceable to the DCCT standard.

\section{Statistical Analysis}

All analyses followed the intention-to-treat principle. To analyze HEI scores, we used linear mixed models with a 
Table 1 Demographic Characteristics of Participants

\begin{tabular}{|c|c|c|c|}
\hline & \multirow{2}{*}{$\begin{array}{l}\text { Immediate receipt of meals } \\
(n=20) \\
\% \text { or mean }(\mathrm{SD})\end{array}$} & \multirow{2}{*}{$\begin{array}{l}\text { Delayed receipt of meals } \\
(n=22)\end{array}$} & \multirow[t]{2}{*}{$p$ value } \\
\hline & & & \\
\hline Age, years & $57.66(12.25)$ & $59.21(13.11)$ & 0.69 \\
\hline Female, \% & 65.00 & 72.73 & 0.74 \\
\hline Race/ethnicity, $\%$ & & & 0.83 \\
\hline Non-Hispanic white & 50.00 & 59.09 & \\
\hline Non-Hispanic black & 25.00 & 27.27 & \\
\hline Hispanic & 20.00 & 13.64 & \\
\hline Other & 5.00 & 0.00 & \\
\hline Education, \% & & & 0.35 \\
\hline$<$ HS diploma & 0.00 & 13.64 & \\
\hline HS diploma & 25.00 & 22.73 & \\
\hline$>$ HS diploma & 75.00 & 63.64 & \\
\hline Income as percent of federal poverty ${ }^{\&}$ (median) & 108.56 & 170.38 & 0.16 \\
\hline Insurance, $\%$ & & & 0.42 \\
\hline Private & 15.00 & 13.64 & \\
\hline Medicare & 10.00 & 0.00 & \\
\hline Medicaid & 20.00 & 36.36 & \\
\hline Dual & 55.00 & 50.00 & \\
\hline Adequate health literacy (NVS > 3), \% & 45.00 & 27.27 & 0.34 \\
\hline Born outside the USA, $\%$ & 40.00 & 22.73 & 0.32 \\
\hline Duration of diabetes, years & $15.29(9.36)$ & $14.17(10.84)$ & 0.72 \\
\hline Hypoglycemia in last 3 months, \% & 35.00 & 71.43 & 0.03 \\
\hline Food insecure in last 30 days, $\%$ & 80.00 & 71.43 & 0.72 \\
\hline Cost-related medication underuse, $\%$ & 30.00 & 27.27 & 1.00 \\
\hline Food and diabetes medication/supplies trade-offs, \% & 21.05 & 45.45 & 0.19 \\
\hline SNAP participation in last 12 months, $\%$ & 65.00 & 68.18 & 1.00 \\
\hline Self-reported health status "excellent or very good," \% & 55.00 & 54.55 & 1.00 \\
\hline Health-related quality of life - physical health* & $13.15(13.09)$ & $10.50(10.79)$ & 0.48 \\
\hline Health-related quality of life - mental health* & $6.75(11.57)$ & $13.64(13.43)$ & 0.08 \\
\hline Health-related quality of life-health interference* & $10.80(13.05)$ & $7.95(10.10)$ & 0.44 \\
\hline Diabetes distress score ${ }^{\wedge}$ & $34.45(14.67)$ & $33.86(10.93)$ & 0.89 \\
\hline PHQ-8 score \# $^{\#}$ & $6.05(4.52)$ & $8.14(4.98)$ & 0.17 \\
\hline History of hypertension, $\%$ & 92.86 & 93.33 & 1.00 \\
\hline History of coronary heart disease, $\%$ & 28.57 & 26.67 & 1.00 \\
\hline History of congestive heart failure, $\%$ & 14.29 & 26.67 & 0.65 \\
\hline History of depression, $\%$ & 7.14 & 40.00 & 0.08 \\
\hline Charlson comorbidity score, $\%$ & $4.21(2.89)$ & $5.40(3.07)$ & 0.29 \\
\hline Pre-study HEI total (range $0-100$ ) & $57.27(14.65)$ & $57.16(13.49)$ & 0.98 \\
\hline HEI 1: total vegetables (range $0-5$ ) & $3.01(1.88)$ & $3.03(1.63)$ & 0.98 \\
\hline HEI 2: greens and beans (range $0-5$ ) & $1.99(2.42)$ & $2.29(2.41)$ & 0.70 \\
\hline HEI 3: total fruit (range $0-5$ ) & $2.48(2.25)$ & $3.07(2.12)$ & 0.39 \\
\hline HEI 4: whole fruit (range $0-5$ ) & $2.16(2.27)$ & $3.00(2.35)$ & 0.25 \\
\hline HEI 5: wholegrain (range $0-10$ ) & $2.92(2.71)$ & $3.29(3.84)$ & 0.72 \\
\hline HEI 6: total dairy (range $0-10$ ) & $5.04(3.63)$ & $4.78(3.48)$ & 0.81 \\
\hline HEI 7: total protein (range $0-5$ ) & $4.67(0.93)$ & $4.45(1.30)$ & 0.54 \\
\hline HEI 8: seafood and plant protein (range $0-5$ ) & $2.61(2.46)$ & $1.91(2.30)$ & 0.35 \\
\hline HEI 9: fatty acids (range $0-10$ ) & $6.03(3.47)$ & $5.83(3.58)$ & 0.86 \\
\hline HEI 10: sodium (range $0-10$ ) & $3.83(4.04)$ & $3.37(3.47)$ & 0.69 \\
\hline HEI 11: refined grain (range $0-10$ ) & $6.39(3.23)$ & $6.56(3.59)$ & 0.87 \\
\hline HEI 12: "empty" calories (range 0-20) & $16.14(4.75)$ & $15.59(5.13)$ & 0.72 \\
\hline Baseline hemoglobin A1c, \% & $8.57(1.72)$ & $7.82(1.23)$ & 0.12 \\
\hline Baseline low-density lipoprotein cholesterol, mg/dL & $101.89(45.76)$ & $109.18(29.81)$ & 0.56 \\
\hline Baseline total cholesterol, $\mathrm{mg} / \mathrm{dL}$ & $177.42(50.08)$ & $181.73(36.35)$ & 0.76 \\
\hline Baseline high-density lipoprotein cholesterol, mg/dL & $47.26(12.93)$ & $45.50(8.57)$ & 0.62 \\
\hline Baseline triglycerides, $\mathrm{mg} / \mathrm{dL}$ & $141.58(86.25)$ & $135.45(65.98)$ & 0.80 \\
\hline Baseline systolic blood pressure, $\mathrm{mm} \mathrm{Hg}$ & $135.25(26.04)$ & $132.16(14.32)$ & 0.64 \\
\hline Baseline diastolic blood pressure, $\mathrm{mm} \mathrm{Hg}$ & $79.38(11.30)$ & $77.52(11.52)$ & 0.60 \\
\hline Baseline body mass index, $\mathrm{kg} / \mathrm{m}^{2}$ & $34.48(5.70)$ & $35.69(7.41)$ & 0.56 \\
\hline
\end{tabular}

HEI, Health Eating Index; NVS, newest vital sign; *range is 0-30, lower score is better; ^range is 17-102, lower is better; ${ }^{\#}$ Patient Health Questionnaire 8, range 0-24, lower is better; ' based on 2016 federal poverty guideline: https://aspe.hhs.gov/computations-2016-poverty-guidelines. HEI subscales are scored such that a high score always indicates "healthier" consumption. For subscales 1-8, a higher score represents higher consumption. For subscales 9-12, guidelines emphasize that these dietary components should be consumed in moderation; thus, a higher score indicates lower consumption

participant-level random effects term to account for repeated assessments. The primary comparison was between "onmeals" and "off-meals" HEI scores, allowing participants to serve as their own controls. Models were adjusted for prestudy HEI score, and study arm to account for any effect of the order in which the intervention was received. The pre-study HEI was a one-time assessment conducted when participants knew they were attending a study visit for dietary assessment. Therefore, it might not accurately represent their usual diet. For this reason, in our pre-specified analysis plan, we chose to 
use this only as an adjustment variable, rather than calculating "change from baseline" scores. Rather, we specified that the "on-meal" and "off-meal" scores, which included multiple unscheduled assessments, were the contrast of interest. We conducted sensitivity analyses that tested for an interaction between the order in which the intervention was received and the HEI score in the "on-meals" compared with "off-meals" period. To analyze secondary outcomes, we accounted for repeated measures with generalized estimating equations. A two-sided $p$ value $<0.05$ was considered statistically significant. With these analysis specifications, and assuming retention of at least 40 participants, we calculated that the study had greater than $80 \%$ power to detect a difference of 5 points or more in the total HEI score. Study data were collected and managed using REDCap (Research Electronic Data Capture) tools hosted at Partners HealthCare. All analyses were conducted using SAS 9.4 (SAS institute, Cary, NC).

\section{RESULTS}

Forty-four participants enrolled in the study. Two participants withdrew from the study, one prior to beginning the study, citing time constraints, and one after receiving 1 week of food delivery, citing gastrointestinal symptoms (nausea/vomiting and diarrhea). Forty-one participants were recruited from within the main primary care network, and three were referred in from outside the main network. We completed telephone follow-up for primary outcome assessment in all 42 remaining study participants. In-person follow-up for secondary outcome assessment was completed for 39 of 42 "off-meal" periods and 31 of 42 "on-meal" periods (Fig. 1). The mean age of study participants was 58.5 (SD 12.6) years, 69\% were women, and $54 \%$ were non-Hispanic white race/ethnicity (Table 1). Thirtyone percent had high school diploma or less education, and the median income level was $140 \%$ of the federal poverty level. Mean baseline BMI was 35.1 (SD 6.6) $\mathrm{kg} / \mathrm{m}^{2}$, and mean baseline $\mathrm{HbAlc}$ was $8.2 \%$ [66 $\mathrm{mmol} / \mathrm{mol}$ ] (SD $1.5 \%$ $[16.4 \mathrm{mmol} / \mathrm{mol}])$. The cost of the meal program (meals and delivery) was approximately $\$ 350$ per individual per month.

Participants experienced significant improvements in total HEI score while receiving meals (Table 2). Mean "on-meal" total HEI score was 71.3 (SD 7.5) while mean "off-meal" HEI score was 39.9 (SD 7.8), for a difference of 31.4 points $(p<0.0001)$. Participants experienced improvements in almost all sub-categories of HEI score, most notably for increases in consumption of vegetables, fruits, and whole grains, and decreases in "empty" calories from solid fats, alcohol, and added sugars. We did not observe an interaction between order of receiving the intervention (immediate versus delayed) and total HEI score during the "on-meals" vs. "offmeals" period. The difference in mean HEI score between "on-meals" and "off-meals" periods for those who received the intervention first was 30.5 points, and the difference in mean HEI score between "on-meals" and "off-meals" periods for those who received the intervention after a waiting period was 32.3 points ( $p$ for interaction $=0.57$ ).

Participants reported large reductions in food insecurity, with $42 \%$ reporting food insecurity during "on-meal" vs. $62 \%$ during "off-meal" period, $p=0.047$. Participants also reported reductions in hypoglycemia, with $47 \%$ reporting hypoglycemia during "on-meal" vs. 64\% during "off-meal" period, $p=0.03$, along with improvements in the mental health component of health-related quality of life (Table 3). Exploratory outcomes, which, as expected, did not show significant changes during the short study period, are presented in Table 4.

One patient reported gastrointestinal symptoms during the "on-meals" period. No other study-related adverse events were reported.

Table 2 Differences in Healthy Eating Index (HEI) Total Score and Subscores at the End of the "On-Meals" and "Off-Meals" Period with Participants Serving as Their Own Controls per the Randomized Cross-over Design

\begin{tabular}{|c|c|c|c|}
\hline Variable & $\begin{array}{l}\text { On-meals } \\
\text { Mean (SD) }\end{array}$ & $\begin{array}{l}\text { Off-meals } \\
\text { Mean (SD) }\end{array}$ & $p$ value \\
\hline HEI total (range $0-100)$ & $71.30(7.49)$ & $39.89(7.80)$ & $<0.0001$ \\
\hline HEI 1: total vegetables (range $0-5$ ) & $4.45(0.63$ & $2.51(1.01)$ & $<0.0001$ \\
\hline HEI 2: greens and beans (range $0-5$ ) & $3.83(1.32)$ & $0.79(1.09)$ & $<0.0001$ \\
\hline HEI 3: total fruit (range $0-5$ ) & $3.53(1.26)$ & $1.32(1.12)$ & $<0.0001$ \\
\hline HEI 4: whole fruit (range 0-5) & $3.88(1.27)$ & $1.13(1.11)$ & $<0.0001$ \\
\hline HEI 5: wholegrain (range $0-10$ ) & $5.22(2.58)$ & $1.74(1.60)$ & $<0.0001$ \\
\hline HEI 6: total dairy (range $0-10$ ) & $5.39(2.37)$ & $4.75(1.92)$ & 0.17 \\
\hline HEI 7: total protein (range $0-5$ ) & $4.85(0.52)$ & $3.77(1.03)$ & $<0.0001$ \\
\hline $\begin{array}{l}\text { HEI 8: seafood and plant protein } \\
\text { (range } 0-5 \text { ) }\end{array}$ & $3.47(1.22)$ & $1.39(1.19)$ & $<0.0001$ \\
\hline HEI 9: fatty acids (range $0-10$ ) & $7.80(1.88)$ & $4.63(2.15)$ & $<0.0001$ \\
\hline HEI 10: sodium (range $0-10$ ) & $1.56(1.41)$ & $3.17(1.92)$ & 0.0002 \\
\hline $\begin{array}{l}\text { HEI 11: refined grain } \\
\text { (range } 0-10)\end{array}$ & $9.07(1.68)$ & $5.78(2.39)$ & $<0.0001$ \\
\hline HEI 12: "empty" calories (range 0-20) & $18.25(2.19)$ & $8.89(3.52)$ & $<0.0001$ \\
\hline
\end{tabular}

HEI, Health Eating Index. HEI subscales are scored such that a high score always indicates "healthier" consumption. For subscales 1-8, a higher score represents higher consumption. For subscales 9-12, guidelines emphasize that these dietary components should be consumed in moderation; thus, a higher score indicates lower consumption 
Table 3 Participant-Reported Outcomes

\begin{tabular}{|c|c|c|c|}
\hline & \multirow{2}{*}{$\frac{\text { On-Meals }}{N=31}$} & \multirow{2}{*}{$\frac{\text { Off-Meals }}{\bar{N}=39}$} & \multirow[t]{2}{*}{$p$ value } \\
\hline & & & \\
\hline Percent reporting food insecurity & 41.94 & 61.54 & 0.047 \\
\hline Percent reporting hypoglycemia in prior 3 months & 46.67 & 63.89 & 0.03 \\
\hline Percent reporting health status "excellent or very good" & 54.84 & 48.72 & 0.50 \\
\hline Health-related quality of life-physical health* & 13.87 & 12.05 & 0.50 \\
\hline Health-related quality of life-mental health* & 5.65 & 9.59 & 0.03 \\
\hline Health-related quality of life-health interference* & 8.19 & 7.87 & 0.89 \\
\hline Diabetes distress $\wedge$ & 30.94 & 30.82 & 0.96 \\
\hline Percent reporting cost-related medication underuse & 22.58 & 28.21 & 0.52 \\
\hline Percent reporting food-medication trade-offs in prior 3 months & 29.03 & 23.08 & 0.12 \\
\hline PHQ-8 score ${ }^{\&}$ & 6.16 & 6.13 & 0.96 \\
\hline
\end{tabular}

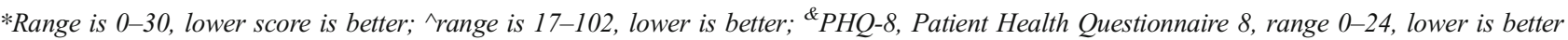

\section{DISCUSSION}

In this randomized cross-over clinical trial, home delivery of medically tailored meals to food-insecure individuals with diabetes substantially improved dietary quality, improved food insecurity, and reduced hypoglycemia. Medically tailored meals were also acceptable to participants. To put the change in dietary quality in context, nationally representative data from the USA indicates that the mean HEI for adults is 49.6, with an important gap between those with low socioeconomic status (mean 44.9) and high socioeconomic status (mean 53.1). ${ }^{21}$ In this study, the "off-meal" HEI score was lower than this mean, while the "onmeal" was higher, illustrating the magnitude of improvement seen during the intervention. Prior studies have shown that sustained differences in dietary quality of a similar magnitude to that seen here, albeit using a different index of dietary quality, have been associated with $41 \%$ reduction in all-cause mortality in men with type 2 diabetes. ${ }^{22}$

While it seems logical that a direct feeding program would improve dietary quality, this had not been demonstrated previously in the context of type 2 diabetes. Because direct feeding programs inherently reduce food choice and may suffer from issues of cultural acceptability or palatability, it was possible that the program would not be accepted by the participants. Further, since the intervention provided only $50 \%$ of meals, consumption of less healthy food outside the program could have undermined any change in dietary quality. The results of this study show, however, that the intervention was not affected by these concerns. Prior, non-randomized, pre/post studies of medically tailored meals ${ }^{23}$ and food pantrybased interventions ${ }^{24}$ have also suggested benefit of the direct food provision, but the use of random assignment in this study strengthens the evidence base to support a causal effect between the medically tailored meal program and improved dietary quality.

This study is consistent with and expands our knowledge regarding food insecurity interventions in individuals with diabetes. Food insecurity is a problem of both food access and food utilization. The United Nation's Food and Agriculture Organization considers food security to include both economic food access (the ability to afford food appropriate to one's needs) and food utilization (the ability to use the food available considering cultural acceptability, time and equipment needed to prepare it, knowledge, and taste preferences). ${ }^{25}$ The most common intervention strategies in the USA are income-transfer programs (like the Supplemental Nutrition Assistance Program [SNAP]), which provide economic resources to improve food access. Prior work has suggested that SNAP may be associated with reduced health care expenditures among American adults. ${ }^{26-28}$ The intervention studied is of a different type - a food provision program tailored to the

Table 4 Exploratory Biometric Outcomes

\begin{tabular}{|c|c|c|c|c|c|}
\hline & \multicolumn{2}{|c|}{ On-meals } & \multicolumn{2}{|c|}{ Off-meals } & \multirow[t]{2}{*}{$p$ value } \\
\hline & Mean & SD & Mean & SD & \\
\hline Hemoglobin A1c, \% & 7.99 & 1.86 & 8.15 & 1.72 & 0.57 \\
\hline Low-density lipoprotein cholesterol, mg/dL & 97.54 & 35.67 & 101.43 & 43.11 & 0.93 \\
\hline Total cholesterol, mg/dL & 182.63 & 47.30 & 178.69 & 43.82 & 0.67 \\
\hline High-density lipoprotein cholesterol, mg/dL & 45.81 & 11.23 & 46.36 & 10.20 & 0.59 \\
\hline Systolic blood pressure, $\mathrm{mm} \mathrm{Hg}$ & 133.04 & 17.60 & 136.00 & 18.77 & 0.39 \\
\hline Diastolic blood pressure, $\mathrm{mm} \mathrm{Hg}$ & 76.86 & 8.65 & 78.16 & 10.11 & 0.29 \\
\hline Baseline body mass index, $\mathrm{kg} / \mathrm{m}^{2}$ & 34.24 & 6.76 & 34.79 & 6.97 & 0.93 \\
\hline Triglycerides, $\mathrm{mg} / \mathrm{dL}$ & $142.00 *$ & $\mathrm{n} / \mathrm{a}$ & $127.50 *$ & $\mathrm{n} / \mathrm{a}$ & $0.16^{* *}$ \\
\hline
\end{tabular}

*Values presented are median owing to highly non-normal distribution; **p value from non-parametric signed rank test [for paired non-normally distributed data]

These were exploratory outcomes, and the trial was not designed or powered to test hypotheses regarding changes in these outcomes 
medical needs of participants. Food provision programs can improve both food access and food utilization by overcoming further barriers that income-transfer programs may not address, such as insufficient time or knowledge to prepare healthy meals ${ }^{29}$ or choosing to purchase foods less appropriate to manage diabetes, particularly if healthier foods are less palatable and putative future health benefits are not as salient when in difficult financial circumstances. ${ }^{30}$ The medically tailored meal program helped make healthy, prepared foods readily available. Prior studies have observed that food insecurity is associated with worse mental health. ${ }^{31}$ This study found that the mental health component of health-related quality of life improved when "on-meals," although we caution that this was a secondary study outcome.

This study included socioeconomically vulnerable and racially/ethnically diverse individuals who have historically been underrepresented in studies of diabetes interventions and in clinical research in general, in part due to significant barriers to participation, some of which were evident in this trial. In particular, detailed and rigorous in-person assessments were essential in order to maximize internal validity, but asking participants who often face significant time constraints and transportation barriers to come in for study visits likely limited the number who were able to participate. Since the intervention concept was unproven, we believe an "efficacy"-type design which emphasized internal validity, potentially at the expense of generalizability, was appropriate as a first step. Going forward, we believe that future work should emphasize a pragmatic or "effectiveness" approach in order to enhance the external validity of future findings. Notably, the intervention itself (home delivery of meals), as separate from the procedures necessary to study it in this instance, helps overcome many barriers to participation.

As would be expected in a sample of this study's size, there was some residual imbalance of covariates between the immediate and delayed intervention groups, as seen in Table 1. However, because of the cross-over design of the trial, the comparison is performed within individuals at different times (that is, when they are receiving and not receiving meals), rather than between the immediate and delayed intervention groups. Therefore, these differences do not affect the interpretation of the results.

Results from this study should be interpreted in light of several limitations. This was a short-term study designed to test whether a medically tailored meal program could change dietary quality in a way that would be expected to lead to clinical improvements in a larger-scale study. While adequate for this purpose, results should not be extrapolated to longer time frames or larger groups at this time. Additionally, while follow-up for the primary outcome was adequate, there was loss to in-person follow-up for some secondary outcomes, which may have reduced power, or, if loss to follow-up was differential, affected the results. To help mitigate this, however, all analyses were conducted using the intention-to-treat principle to preserve randomization. Neither participants nor study staff were masked to intervention assignment. Owing to high respondent burden, we did not directly track adherence to meals. The proportion of women included in the study was higher than that of the primary care network from which participants were recruited. These limitations are balanced by several strengths. These include the enrollment of a racial/ ethnically diverse and socioeconomically vulnerable sample who are often left out of studies of diabetes interventions. ${ }^{32}$ Follow-up for the primary outcome was excellent, and the randomized design and detailed in-person assessments enhance the internal validity of the findings. Overall, the study population was representative of the primary care population from which it was drawn. ${ }^{33}$ Finally, the cross-over design provided additional control for unmeasured, time-invariant factors, as participants were being compared with themselves in different time periods.

This study has important implications for future work. The promising results in this short-term study justify longer-term studies to elucidate the impact of medically tailored meal programs on measures of diabetes control, along with clinical outcomes such as reductions in hypoglycemia and use of healthcare services. The findings also figure into larger national discussions regarding the best way for clinical care organizations to address social determinants of health. In the Accountable Health Communities model from the Centers for Medicare and Medicaid Services, food insecurity is a priority area for screening, but the optimal interventional strategy for those who screen positive remains unclear. ${ }^{34}$ Currently, medically tailored meals are a covered service under the Ryan White HIV/AIDS Program and under recent changes to the Medicare Advantage program. ${ }^{35}$ They are not covered in larger federal programs such as traditional Medicare or Medicaid fee-for-service programs. Should the value of medically tailored meals be demonstrated $^{36}$, medically tailored meals might be included as a covered benefit under particular circumstances where the program has been shown to be of benefit.

Medically tailored meal programs show promise for improving dietary quality and reducing food insecurity and hypoglycemia in individuals with diabetes. We believe longerterm studies are warranted. These should study the effects of medically tailored meal programs on diabetes control and well-being among individuals with diabetes and food insecurity. If these studies are successful, medically tailored meal programs may be an important way to improve health for vulnerable individuals with diabetes.

\section{Acknowledgments:}

The authors thank the participants of the study and the Diabetes Research Center at Massachusetts General Hospital for serving as the study site.

Author Information: $S A B$ and DJW conceived of the study and drafted the manuscript. JTand LMD conceived of the study and revised the manuscript critically for intellectual content. BS, MR, RS, and NS assisted with collection and interpretation of the data and revised the manuscript critically for intellectual content. All authors give approval of the manuscript version to be submitted. 
Corresponding Author: Seth A. Berkowitz, MD MPH; Division of General Medicine and Clinical Epidemiology, Department of Medicine University of North Carolina School of Medicine, Chapel Hill, NC, USA (e-mail: seth_berkowitz@med.unc.edu).

Funding Information Funding for this study was provided by the Blue Cross Blue Shield Foundation of Massachusetts, BNY Mellon, the Diabetes Research Center and Division of General Internal Medicine at Massachusetts General Hospital, and the National Institute of Diabetes and Digestive and Kidney Diseases of the National Institutes of Health under Award Number K23DK109200. The content is solely the responsibility of the authors and does not necessarily represent the official views of the National Institutes of Health.

\section{Compliance with Ethical Standards:}

Conflict of Interest: Jean Terranova is an employee of Community Servings. All other authors declare they have nothing to disclose.

Prior Presentation: An interim version of these findings was reported at the 2017 Society for General Internal Medicine annual meeting, in Washington, DC.

Guarantor: Seth A. Berkowitz had full access to all of the data in the study and takes full responsibility for the work as a whole, including the study design, access to data, the integrity of the data, the accuracy of the data analysis, and the decision to submit and publish the manuscript.

\section{REFERENCES}

1. Centers for Disease Control and Prevention. Diabetes Fact Sheets. https://www.cdc.gov/diabetes/library/factsheets.html. Published December 26, 2017. Accessed 17 Sep 2018.

2. Association AD. 1. Improving Care and Promoting Health in Populations: Standards of Medical Care in Diabetes-2018. Diabetes Care. 2018;41(Supplement 1):S7-S12. doi:https://doi.org/10.2337/dc18$\mathrm{S} 001$

3. Bickel G, Nord M, Price C, Hamilton W, Cook J. Guide to Measuring Household Food Security, Revised 2000. March 2000. https://fns-prod. azureedge.net/sites/default/files/FSGuide.pdf. Accessed 17 Sep 2018.

4. Berkowitz SA, Baggett TP, Wexler DJ, Huskey KW, Wee CC. Food insecurity and metabolic control among U.S. adults with diabetes. Diabetes Care. 2013;36(10):3093-3099. doi:https://doi.org/10.2337/ dc13-0570

5. Seligman HK, Laraia BA, Kushel MB. Food insecurity is associated with chronic disease among low-income NHANES participants. J Nutr. 2010;140(2):304-310. doi:https://doi.org/10.3945/jn.109.112573

6. American Diabetes Association. 6. Glycemic Targets. Diabetes Care. 2017;40(Supplement 1):S48-S56. doi:https://doi.org/10.2337/dc17-S009

7. Seligman HK, Schillinger D. Hunger and socioeconomic disparities in chronic disease. N Engl J Med. 2010;363(1):6-9. doi:https://doi.org/10. 1056/NEJMp1000072

8. Berkowitz SA, Gao X, Tucker KL. Food-insecure dietary patterns are associated with poor longitudinal glycemic control in diabetes: results from the Boston Puerto Rican Health study. Diabetes Care. 2014;37(9):2587-2592. doi:https://doi.org/10.2337/dc14-0753

9. Basu S, Berkowitz SA, Seligman H. The Monthly Cycle of Hypoglycemia: an Observational Claims-based Study of Emergency Room Visits, Hospital Admissions, and Costs in a Commercially Insured Population. Med Care. 2017;55(7):639-645. doi:https://doi.org/10.1097/MLR 0000000000000728

10. Seligman HK, Davis TC, Schillinger D, Wolf MS. Food insecurity is associated with hypoglycemia and poor diabetes self-management in a low-income sample with diabetes. J Health Care Poor Underserved. 2010;21(4):1227-1233. doi:https://doi.org/10.1353/hpu.2010.0921

11. Seligman HK, Jacobs EA, Lopez A, Sarkar U, Tschann J, Fernandez A. Food insecurity and hypoglycemia among safety net patients with diabetes. Arch Intern Med. 2011;171(13):1204-1206. doi:https://doi. org/10.1001/archinternmed.2011.287
12. Seligman HK, Bolger AF, Guzman D, López A, Bibbins-Domingo K. Exhaustion of food budgets at month's end and hospital admissions for hypoglycemia. Health Aff Proj Hope. 2014;33(1):116-123. doi:https://doi. org/10.1377/hlthaff.2013.0096

13. Coppell KJ, Kataoka M, Williams SM, Chisholm AW, Vorgers SM, Mann JI. Nutritional intervention in patients with type 2 diabetes who are hyperglycaemic despite optimised drug treatment-Lifestyle Over and Above Drugs in Diabetes (LOADD) study: randomised controlled trial. BMJ. 2010;341:c3337.

14. Look AHEAD Research Group, Wing RR. Long-term effects of a lifestyle intervention on weight and cardiovascular risk factors in individuals with type 2 diabetes mellitus: four-year results of the Look AHEAD trial. Arch Intern Med. 2010;170(17):1566-1575. doi:https://doi.org/10.1001/ archinternmed.2010.334

15. Gottlieb LM, Wing H, Adler NE. A Systematic Review of Interventions on Patients' Social and Economic Needs. Am J Prev Med. July 2017. doi:https://doi.org/10.1016/j.amepre.2017.05.011

16. Hager ER, Quigg AM, Black MM, et al. Development and validity of a 2item screen to identify families at risk for food insecurity. Pediatrics. 2010;126(1):e26-32. doi:https://doi.org/10.1542/peds.2009-3146

17. Gundersen C, Engelhard EE, Crumbaugh AS, Seligman HK. Brief assessment of food insecurity accurately identifies high-risk US adults. Public Health Nutr. 2017;20(8):1367-1371. doi:https://doi.org/10. $1017 / \mathrm{S} 1368980017000180$

18. Center for Nutrition Policy and Promotion. Healthy Eating Index (HEI). https://www.cnpp.usda.gov/healthyeatingindex. Accessed 17 Sep 2018.

19. Schwingshackl L, Bogensberger B, Hoffmann G. Diet Quality as Assessed by the Healthy Eating Index, Alternate Healthy Eating Index, Dietary Approaches to Stop Hypertension Score, and Health Outcomes: an Updated Systematic Review and Meta-Analysis of Cohort Studies. J Acad Nutr Diet. 2018;118(1):74-100.e11. doi:https://doi.org/10.1016/j. jand.2017.08.024

20. HEI Scores for Examining the Effect of an Intervention. https://epi. grants.cancer.gov/hei/hei-scores-examining-effect-of-intervention.html. Accessed 17 Sep 2018.

21. Wang DD, Leung CW, Li Y, et al. Trends in Dietary Quality Among Adults in the United States, 1999 Through 2010. JAMA Intern Med. 2014;174(10):1587-1595. doi:https://doi.org/10.1001/jamainternmed. 2014.3422

22. Patel YR, Gadiraju TV, Gaziano JM, Djoussé L. Adherence to healthy lifestyle factors and risk of death in men with diabetes mellitus: the Physicians' Health Study. Clin Nutr Edinb Scotl. November 2016. https://doi.org/10.1016/j.clnu.2016.11.003

23. Palar K, Napoles T, Hufstedler LL, et al. Comprehensive and Medically Appropriate Food Support Is Associated with Improved HIV and Diabetes Health. J Urban Health Bull N Y Acad Med. 2017;94(1):87-99. doi:https://doi.org/10.1007/s11524-016-0129-7

24. Seligman HK, Lyles C, Marshall MB, et al. A Pilot Food Bank Intervention Featuring Diabetes-Appropriate Food Improved Glycemic Control Among Clients In Three States. Health Aff Proj Hope. 2015;34(11):1956-1963. doi:https://doi.org/10.1377/hlthaff.2015.0641

25. United Nations Food and Agriculture Organization. An Introduction to the Basic Concepts of Food Security. 2008. http://www.fao.org/docrep/ 013/al936e/al936e00.pdf. Accessed 17 Sep 2018.

26. Berkowitz SA, Seligman HK, Rigdon J, Meigs JB, Basu S. Supplemental Nutrition Assistance Program (SNAP) Participation and Health Care Expenditures Among Low-Income Adults. JAMA Intern Med. 2017;177(11):1642-1649. doi:https://doi.org/10.1001/jamainternmed. 2017.4841

27. Szanton SL, Samuel LJ, Cahill R, et al. Food assistance is associated with decreased nursing home admissions for Maryland's dually eligible older adults. BMC Geriatr. 2017;17(1):162. doi:https://doi.org/10.1186/ s 12877-017-0553-X

28. Samuel LJ, Szanton SL, Cahill R, et al. Does the Supplemental Nutrition Assistance Program Affect Hospital Utilization Among Older Adults? The Case of Maryland Popul Health Manag. July 2017. https:// doi.org/10.1089/pop.2017.0055

29. Monsivais P, Aggarwal A, Drewnowski A. Time spent on home food preparation and indicators of healthy eating. Am J Prev Med. 2014;47(6):796-802. doi:https://doi.org/10.1016/j.amepre.2014.07. 033

30. Epstein LH, Jankowiak N, Lin H, Paluch R, Koffarnus MN, Bickel WK. No food for thought: moderating effects of delay discounting and future time perspective on the relation between income and food insecurity. Am J Clin Nutr. 2014;100(3):884-890. doi:https://doi.org/10.3945/ajcn. 113.079772 
31. Silverman J, Krieger J, Kiefer M, Hebert P, Robinson J, Nelson K. The Relationship Between Food Insecurity and Depression, Diabetes Distress and Medication Adherence Among Low-Income Patients with PoorlyControlled Diabetes. J Gen Intern Med. 2015;30(10):1476-1480. doi:https://doi.org/10.1007/s11606-015-3351-1

32. Spong CY, Bianchi DW. Improving Public Health Requires Inclusion of Underrepresented Populations in Research. JAMA. 2018;319(4):337. doi:https://doi.org/10.1001/jama.2017.19138

33. Berkowitz SA, Meigs JB, DeWalt D, et al. Material need insecurities, control of diabetes mellitus, and use of health care resources: results of the Measuring Economic Insecurity in Diabetes study. JAMA Intern Med.
2015;175(2):257-265. doi:https://doi.org/10.1001/jamainternmed. 2014.6888

34. Alley DE, Asomugha CN, Conway PH, Sanghavi DM. Accountable Health Communities-Addressing Social Needs through Medicare and Medicaid. N Engl J Med. 2016;374(1):8-11. doi:https://doi.org/10.1056/ NEJMp1512532

35. Willink A, DuGoff EH. Integrating Medical and Nonmedical Services The Promise and Pitfalls of the CHRONIC Care Act. N Engl J Med. 2018;378(23):2153-2155. doi:https://doi.org/10.1056/NEJMp1803292

36. Berkowitz SA, Terranova J, Hill C, et al. Meal Delivery Programs Reduce The Use Of Costly Health Care In Dually Eligible Medicare And 